\title{
Application of Nonresonance Excitation to Ion Trap Tandem Mass Spectrometry and Selected Ejection Chemical Ionization
}

\author{
Mingda Wang, Steve Schachterle, and Greg Wells \\ Varian Chromatography Systems, 2700 Mitchell Drive, Walnut Creek, California, USA
}

\begin{abstract}
Nonresonance excitation is a universal ion excitation and ejection method in which increased ion kinetic energy is achieved by the combination of an axial dc dipole and the $\mathrm{rf}$ trapping fields. The method does not require the applied excitation frequency to match with the secular frequency of the precursor ions to effect collision-induced dissociation (CID) for tandem mass spectrometry applications. Therefore, it is free of the effects of secular frequency changes caused by space-charge and simplifies the optimization of tandem mass spectrometry parameters when combined with gas chromatography-tandem mass spectrometry (GC-MS/MS). Computer simulations show that in contrast to the resonance excitation process, the nonresonance excitation process is able to accelerate thermal ions to kinetic energies in excess of $40 \mathrm{eV}$ in a few microseconds. Based on simulations, we expect that the rapid deposition of energy by this method may allow the study, in ion traps, of high energy decomposition channels of precursor ions with multiple decomposition channels. Furthermore, the method is able to simultaneously excite multiple precursor ions, for example, excite both analyte and its coeluting isotopically labeled internal standard for GC-MS/MS analysis. A GC-MS/MS analysis of $100 \mathrm{pg}$ of $n$-butylbenzene is demonstrated with a signal-to-noise ratio of 3624, which is over an order of magnitude higher than the signal-tonoise ratio of 345 obtained by full scan gas chromatography-mass spectrometry. In addition, the nonresonance excitation method can be used as a low pass mass filter in the chemical ionization (CI) mode to eject undesired fragment ions that result from direct electron ionization. This new CI method, selected ejection chemical ionization, can produce a $\mathrm{CI}$ spectrum without contamination of sample fragment ions from electron ionization. (J Am Soc Mass Spectrom 1996, 7, 668-676)
\end{abstract}

$\mathrm{T}$ he quadrupole ion trap has become increasingly important as a bench-top analytical mass spectrometer. The sensitivity of ion traps [1], the extension of mass range into the tens of thousands of mass units [2-4], the interfacing of external ion sources [5-8], the capability of high mass resolution [9-12], and the ability to perform tandem mass spectrometry [13-15] have enhanced the analytical utility of the ion trap.

Tandem mass spectrometry has been used widely to identify chemical structures, to study reaction mechanisms of ion-neutral reactions, to obtain thermochemical information of gas-phase species, and to improve qualitative and quantitative analysis of complex matrices. Tandem mass spectrometers may be divided into two types of instruments: those tandem in space (e.g., triple quadrupole and multiple sector) and those tandem in time (e.g., Fourier transform ion cyclotron resonance and ion trap). The tandem mass spectrometry method in the quadrupole ion trap typically con-

Addresss reprint requests to Mr. Mingda Wang, Varian Chromatography Systems, 2700 Mitchell Drive, Walnut Creek, CA 94598. sists of three steps. First, the selected precursor ions are isolated in the ion trap. Second, they are dissociated by collision-induced dissociation (CID) and, finally, the product ions are sequentially ejected according to their mass-to-charge ratio from the trap and detected by an electron multiplier.

Currently, most excitation methods for CID in an ion trap are based on resonance excitation that requires the applied excitation frequency to match the secular frequency of the ion motion so that the ions absorb power. The single frequency excitation method used in early tandem quadrupole ion trap mass spectrometers requires a manual tune operation for each individual ion studied, because the ion secular frequency is shifted from its theoretical value due to space-charge and other factors. It is particularly difficult to achieve operation with single frequency resonance activation via gas chromatography, because accurate frequency matching is required in real time as the sample concentration changes during peak elution. To solve the problem of secular frequency mismatching for onresonance excitation, several methods have been proposed: dynamically programmed scans [16], frequency 
assignment pre-scan [17], stored waveform inverse Fourier transform $[18,19]$, band excitation $[17,20]$, noise excitation [21], and single or multiple frequency excitation with modulated rf storage voltage [15]. Boundary activated dissociation $[22,23]$ and fast pulse (for surface-induced dissociation) excitations [24] have been proposed as two alternative methods for resonance excitation, even though product ions yields are reduced. Recently, a dc pulse excitation method has been used for nondestructive detection of ions in a simulation study [25].

In this work we present a universal ion excitation and ejection method for an ion trap. In this method, a low frequency square wave $(50-500 \mathrm{~Hz})$ is differentially applied to the two endcaps via a transformer to create an axial dipolar field (along the $z$ axis of the trap). In practice, use of a transformer has the advantage of creating both endcap voltages with only one amplifier circuit. Use of a transformer also helps to minimize residual endcap voltages caused by the large rf trapping field currents that flow through the endcaps ( $\sim 1 \mathrm{~A}$ in commercial traps). In addition, use of a transformer can remove the dc component to the endcaps. Thus, nonresonance excitation via a transformercoupled low frequency square wave is electronically simpler and more cost effective than use of a dc pulse with direct coupling, and the needed performance is retained.

The new method is independent of the ion mass and the number of ions in the trap [26, 27] because it does not require matching the supplemental excitation frequency with the secular frequency (usually greater than $10 \mathrm{kHz}$ ) of the precursor ions for CID. In the new method, ion energy deposition is achieved by combination of the axial dc dipole and rf trapping fields [26, $27,29]$. When the dipole field turns on, the electrical center of the trapping field and the ion oscillation center are moved almost instantly to a new position [26-28] with no change in the spatial location of the ions. Initially, the ions will have a high potential energy as the result of the perturbation of the trapping field, which is then converted into kinetic energy. The new asymmetrical trapping field will cause the ions to oscillate around the new center. When the ions lose their energy by collision, they may be reexcited by simply turning off or reversing the dipole field. The process may be repeated a number of times if needed to effect high efficiency CID. The method is able to simultaneously excite multiple precursor ions of similar mass, for example, excite both the analyte and its coeluting isotopically labeled internal standard for gas chromatography-tandem mass spectrometry (GCMS/MS) analysis. We demonstrate the application of the new method to the GC-MS/MS analysis of $n$ butylbenzene and tandem mass spectrometry analysis of tetraethylsilane.

Resonance excitation is a low energy deposition process in which the kinetic energy of the ion is increased slowly. This energy is converted to internal energy through multiple collisions with the damping gas. As a result ions tend to decompose predominantly through low energy channels [22]. In this article, computer simulations reveal that nonresonance excitation is able to increase the ion kinetic energy rapidly, within a few microseconds, to values of more than $40 \mathrm{eV}$. Based on simulations, we expect that the rapid deposition of energy by this method will allow the study, in ion traps, of high energy decomposition channels of precursor ions with multiple decomposition channels.

In a traditional chemical ionization source, the residence time of the analyte is less than $100 \mu \mathrm{s}$. Therefore, the traditional chemical ionization source is filled with high pressure reagent gas, $\sim 0.1-1$ torr, to ensure sufficient ion-molecule collisions for effective chemical ionization. Under high pressure, the ratio of reagent gas to analyte is usually about $1000: 1$ so that the ratio of electron ionization (EI) fragment ions to chemical ionization (CI) ions from the analyte effectively is reduced in the $\mathrm{CI}$ mass spectrum. The $\mathrm{CI}$ ion source in ion traps is a low pressure chemical ionization source [30]. Chemical ionization at low pressure is accomplished by utilizing a long reaction period, in the range of 1-100 ms. Because the reagent gas-to-sample ratio is quite low in the low pressure CI source, steps must be taken to reduce the ratio of EI fragment ions to $\mathrm{CI}$ ions of the sample. One means to achieve this is by lowering the rf trapping field during the ionization period [31]. The trapping efficiencies of the higher mass EI fragment ions of sample are reduced significantly with the lower rf trapping field. The trapping efficiency of the lower mass reagent ions is barely affected. During the reaction period, the $\mathrm{rf}$ trapping field is raised and the resulting sample $\mathrm{CI}$ ions effectively are stored in the trap for analysis. Cairns et al. [32] found that under certain conditions, a combined $\mathrm{EI}$ and $\mathrm{CI}$ spectrum was still observed in ion traps. Cairns et al. [33] further demonstrated improved performance by modifying the rf scan function. In this article, we demonstrate that the nonresonance excitation method also provides a means to eject ions with mass-to-charge ratios above a specified value. This effect can be used as a low pass mass filter and can be applied in the CI mode to eject undesired fragment ions that result from direct electron ionization of analytes. This operating mode is called selected ejection chemical ionization (SECI).

\section{Theory}

For simplicity, we shall discuss the effects of nonresonance excitation only under ideal quadrupole and uniform dipole field conditions. In an ideal quadrupole field, the electric potential may be represented by

$$
\phi=\phi_{0}\left(\frac{r^{2}-2 z^{2}}{r_{0}^{2}+2 z_{0}^{2}}+\frac{2 z_{0}^{2}}{r_{0}^{2}+2 z_{0}^{2}}\right)
$$

in which $\phi_{0}=(U-V \cos \Omega t), r_{0}$ is the internal ra- 
dius of the ring electrode, $z_{0}$ is the distance of the endcap tip to the trap center, $\Omega$ is the rf trapping frequency, $U$ is the dc potential applied to the ring electrode, and $V$ is the amplitude of the rf potential applied to the ring electrode. Usually, $r_{0}^{2}=2 z_{0}^{2}$ and eq 1 may be simplified as

$$
\phi=\phi_{0}\left(\frac{r^{2}-2 z^{2}}{2 r_{0}^{2}}+\frac{2 z_{0}^{2}}{2 r_{0}^{2}}\right)
$$

Thus the electric field in the trap is

$$
\begin{aligned}
& E_{z}=-\frac{\partial \phi}{\partial z}=\frac{2 \phi_{0} z}{r_{0}^{2}} \\
& E_{r}=-\frac{\partial \phi}{\partial r}=-\frac{\phi_{0} r}{r_{0}^{2}}
\end{aligned}
$$

in which $E_{z}$ and $E_{r}$ are the $z$ and $r$ components of the trapping electric field, respectively. In the ideal quadrupole field, the electric fields are uncoupled in the $z$ and $r$ directions and, thus, the $z$ and $r$ motions of a charged particle inside the trap are uncoupled.

The equation of ion motion in the $z$ direction is

$$
F_{z}=e E_{z}=m \frac{d^{2} z}{d t^{2}}
$$

in which $m$ and $e$ are ion mass and charge, respectively. Substituting eq 3 into eq 5 and rearranging the resulting equation gives

$$
\frac{d^{2} z}{d t^{2}}-\frac{2 e z}{m r_{0}^{2}} \phi_{0}=0
$$

Equation 6 can be simplified further into the form of a Mathieu equation by substitution of $\phi_{0}=(U-$ $V \cos \Omega t$ ) and $\xi=\Omega t / 2$ into eq 6 and rearrangement,

$$
\frac{d^{2} Z}{d \xi^{2}}+\left(a_{z}-2 q_{z} \cos 2 \xi\right) Z=0
$$

in which $a_{z}=-8 \mathrm{eU} / m r_{0}^{2} \Omega^{2}$ and $q_{z}=-4 \mathrm{eV} / m r_{0}^{2} \Omega^{2}$.

Langmuir and co-workers [28] have derived a simplified approximate solution of ion motion in an ion trap, called the pseudo potential well approximation. In this model the motion in the $z$ direction can be expressed as $z=Z+\delta$. In this approximation, $Z$ describes the average value of the $z$ displacement over a complete period of the rf trapping field and $\delta$ describes the smaller displacements due to the periodically varying if field [28]. For $q_{z}<0.4$, eq 7 may be simplified to

$$
\frac{d^{2} Z}{d \xi^{2}} \approx-\left(a_{z}+\frac{q_{z}^{2}}{2}\right) Z
$$

Equation 8 is a differential equation of harmonic motion and its solution is

$$
Z \approx C_{1} \sin \left(\beta_{z} \xi\right)=C_{1} \sin \left(\frac{1}{2} \beta_{z} \Omega t\right)
$$

in which $\beta_{z} \approx \sqrt{a_{z}+q_{z}^{2} / 2}$ and $\frac{1}{2} \beta_{z} \Omega$ is the ion secular frequency. Equation 9 indicates that under the pseudo potential well approximation, ions oscillate around the trap center $(z=0$ plane) with frequency $\frac{1}{2} \beta_{z} \Omega$.

When a supplementary ac voltage is applied across the two endcap electrodes and its frequency matches the ion secular frequency, the amplitude of ion motion and its kinetic energy increase gradually. If the applied ac electric field is strong enough, ions can absorb enough energy to be ejected from the trap. With a relatively weak supplemental electric field, an ion may collide with the bath gas and convert part of its kinetic energy into internal energy. Under these controlled conditions, ions can undergo multiple collisions and be dissociated. This situation is the basis of CID for all resonance excitation methods.

In this work a low frequency square-wave voltage is applied to the endcaps to create a dipole field along the $z$-axis of the trap. Because the frequency of the square wave is small compared to the secular frequency of the ion, any half cycle can be treated as a constant (dc) dipole field. If a dc dipole field is created by application of a voltage $+V_{D}$ to one endcap and $-V_{\mathrm{D}}$ to the other, the dc dipole may be assumed to be a uniform field with an electric field strength of $2 V_{\mathrm{D}} / 2 z_{0}$ (first order approximation). Under the ideal quadrupole and uniform dipole field approximations, ion motion in the $z$ direction can be described by

$$
\frac{d^{2} z}{d t^{2}}-\frac{2 e z}{m r_{0}^{2}} \phi_{0}=\frac{e}{m} \cdot \frac{2 V_{D}}{2 z_{0}}
$$

Substitution of $\phi_{0}=(U-V \cos \Omega t)$ and $\xi=\Omega t / 2$ into eq 10 and rearrangement allow us to obtain

$$
\frac{d^{2} z}{d \xi^{2}}+\left(a_{z}-2 q_{z} \cos 2 \xi\right) z=\frac{4 e V_{\mathrm{D}}}{z_{0} m \Omega^{2}}
$$

Under the pseudo potential well approximation [28], eq 11 can be simplified to

$$
\frac{d^{2} Z}{d \xi^{2}}+\left(a_{z}+\frac{q_{z}^{2}}{2}\right) Z \approx F
$$

in which $F=4 e V_{\mathrm{D}} / z_{0} m \Omega^{2}$.

The solution of eq 12 is

$$
Z=C_{2} \sin \left(\beta_{z} \xi\right)+\frac{F}{\beta_{z}^{2}}=C_{2} \sin \left(\frac{\beta_{z} \Omega}{2} t\right)+\frac{F}{\beta_{z}^{2}}
$$

Comparing eq 13 with eq 9 , it is clear that superimposition of a uniform dc dipole field onto a pure 
quadrupole field does not change the ion secular frequency $\left(\frac{1}{2} \beta_{z} \Omega\right)$, but the combined fields move the ion oscillation center along the $z$ direction to a new position, $Z=F / \beta_{z}^{2}$.

In the nonresonance excitation method, when the square-wave dipole field turns on, the center of the pseudo potential well is moved instantly to a new position with no change in the spatial location of the ions. The ions convert the potential energy of the trapping field into kinetic energy and oscillate around the new center. Ions may be reexcited simply by turning off or reversing the dipole field. In summary, the main difference between resonance and nonresonance excitation is the time required to impart kinetic energy to the ions. In nonresonance excitation, the ions rapidly increase in kinetic energy as the pseudo potential well is shifted almost instantly by the addition of the dc dipole to the trapping field, whereas, in resonance excitation, kinetic energy increases slowly as the ions are gradually displaced in the fixed trapping field by the supplemental excitation dipole field that is synchronized (in resonance) with the ion motion. It is the nonsynchronous characteristic of the nonresonance excitation that is the basis of its utility.

As shown in eq 13, the pseudo potential well center is displaced to $F / \beta_{z}^{2}$. Under typical ion trap operating conditions, the dc quadrupole field is off and $q_{z} \leq 0.4$. Thus, $a_{z}=0$ and $\beta_{z}^{2} \approx a_{z}+q_{z}^{2} / 2=q_{z}^{2} / 2$. By substitution of $\beta_{z}^{2}=q_{z}^{2} / 2$ and $q_{z}=-4 e V / m r_{0}^{2} \Omega^{2}$ into $F / \beta_{z}^{2}$, we obtain the approximate displacement of ions from the geometrical center of the trap

$$
\Delta=\frac{m V_{D} r_{0}^{4} \Omega^{2}}{2 z_{0} e V^{2}}
$$

For a given ion trap system, $e, r_{0}, \Omega$, and $z_{0}$ are constants and eq 14 may be simplified to

$$
\Delta=C \frac{m V_{\mathrm{D}}}{V^{2}}
$$

in which $C$ is constant. It is clear that the displacement is proportional to the mass of the ion of interest and the amplitude of the applied dc dipole. When $\Delta$ is large enough, the corresponding ions will strike the endcap electrodes or exit from the trap. For a given voltage $V_{\mathrm{D}}$, there is a mass, $m_{\mathrm{c}}$, such that all masses greater than $m_{\mathrm{c}}$ will have $\Delta \mathrm{s}$ large enough to strike the endcap electrodes. Therefore, the dc dipole can be used as a low pass mass filter in the SECI mode to eject ions with mass-to-charge ratios above a specified value.

\section{Results and Discussion}

\section{Model of Nonresonance Excitation}

Figure 1 is the pseudo potential well representation of resonance and nonresonance excitation. In resonance excitation, ions are gradually pushed off the potential

well center by resonance with the supplemental excitation field, as shown at the left side of Figure 1. Thus, ions slowly increase their kinetic energies. In nonresonance excitation the pseudo potential well is instantaneously perturbed by the application of the dipole field. The addition of this field moves the whole pseudo potential well to a new location before ions have time to respond to the movement of the potential well, as shown at the right side of Figure 1. Although ions are still located near the center of the trap electrodes, they are instantly moved away from the center of the electric trapping field to a region of high potential energy. Ions then gain kinetic energy from the trapping field while they oscillate around the new potential well center defined by the combination of the trapping field and the dipole field.

\section{Computer Simulation}

Computer simulations are used to confirm the theoretical prediction given in the previous section. Figure 2 shows the ion trajectory simulation in a nonstretched trap $\left(r_{0}^{2}=2 z_{0}^{2}\right)$ calculated by the SIMION [34] program. In Figure $2 a$, a thermal ion $(0.05 \mathrm{eV})$ of $m / z 134$ is confined to the trap by a rf field with amplitude of $566 \mathrm{~V}_{0-p}$ (zero to peak). As expected, the ion starts to oscillate around the new center immediately after turning on the dc dipole field after $20 \mu \mathrm{s}$. It is worth noting that for an ideal quadrupole and a uniform dc dipole electric field, the ion secular frequency should be independent of the position along the $z$-axis (see eqs 9 and 13). The theoretical treatment given in this work has neglected the effects of the octopole term in the trap-

\section{Resonance}

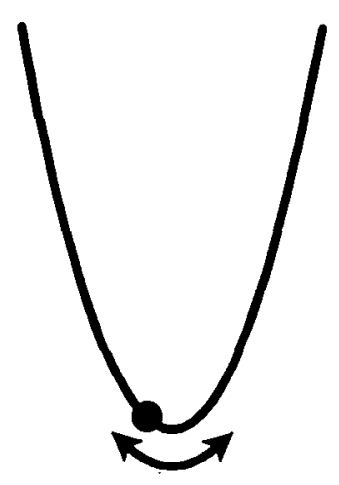

Figure 1. Pseudo potential well representation of resonance and nonresonance excitation. Left: In resonance excitation, ions are slowly displaced from the center of the trapping potential by the force of the external excitation field, which is synchronized to the ion motion. The amplitude of oscillation increases linearly with time. Right: In nonresonance excitation the whole pseudo potential well is moved to a new location (solid line) before ions can follow the movement of the potential well. 


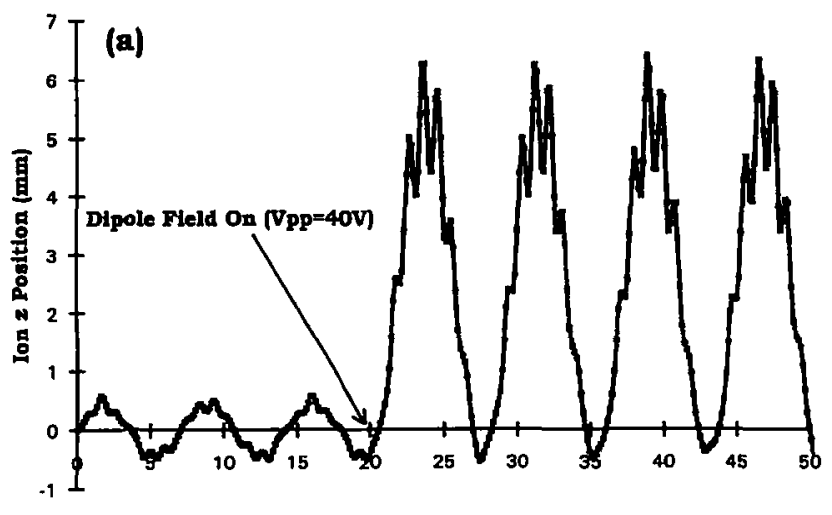

Time (us)

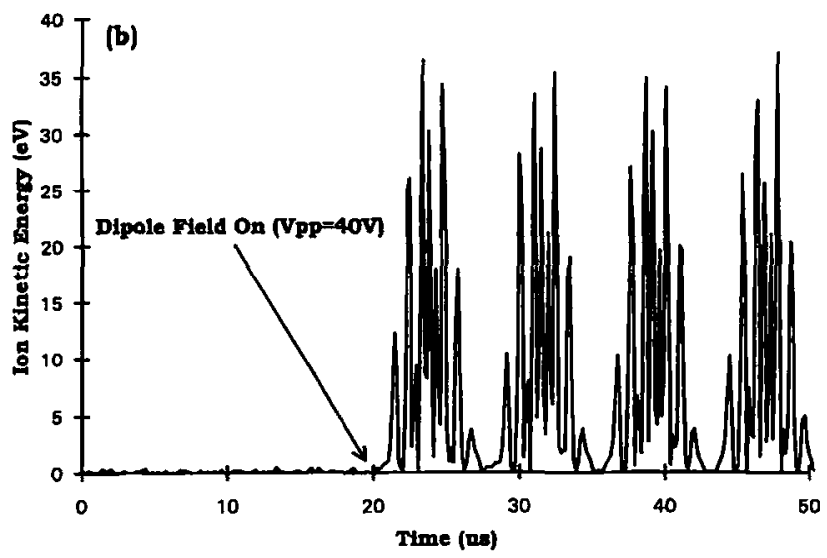

Figure 2. Computer simulation of ion motion calculated by SIMION. (a) The ion motion along the $z$ axis trajectory in a nonstretched trap. A thermal ion $(0.05 \mathrm{eV})$ of $\mathrm{m} / z 134$ is confined to a trap of $r_{0}=1.0 \mathrm{~cm}$ and $z_{0}=0.707 \mathrm{~cm}$ by a $566-\mathrm{V}_{0-\mathrm{p}}$ rf field applied to the ring electrode. The ion starts to oscillate around its new center immediately when a $20-V_{p-p}$ dc dipole is added to the trapping field after $20 \mu \mathrm{s}$. (b) The variation of ion kinetic energy with time before and after nonresonance excitation.

ping field that arises from the truncation of the electrodes. The presence of this term is known to decrease the secular frequency as the amplitude of oscillation is increased [35]. Because the fields in the simulation represent nonideal quadrupole fields, caused by the truncation of the electrodes to a finite length, the calculated ion secular frequency does change when the dc dipole turns on. The decrease in the secular frequency after excitation, due to the increased amplitude, can be seen in Figure 2a. Figure $2 b$ shows how the ion kinetic energy varies with time before and after nonresonance excitation. In contrast to the resonance excitation method, Figure $2 b$ reveals that the nonresonantly excited ion rapidly gains its kinetic energy when the dc dipole field is turned on. A thermal ion of $m / z 134$ in a $40-V_{\text {p-p }}$ (peak to peak) dc dipole field takes only a few microseconds to gain $40-\mathrm{eV}$ translational energy, when confined by a rf field of $566 \mathrm{~V}_{0-\mathrm{p}}$. The maximum ion translational energy can be increased further by increasing the if trapping and $\mathrm{dc}$ dipole fields. Because the operating pressure in ion traps is about $10^{-3}$ torr, each ion will experience a collision approximately every millisecond. This rapid deposition of energy is expected to provide a means to study high energy decomposition channels in ion traps in which there is the competition of low energy decomposition channels [22], which are not feasible with resonance excitation due to the slow increase in kinetic energy. The overall shape of the ion kinetic energy profile (Figure $2 \mathrm{~b}$ ) is similar to the ion displacement shown in Figure 2a and the ion kinetic energy varies rapidly between zero and a maximum. The CID collision energy in the trap depends on the time at which the ion collision occurs. The product ion spectrum in an ion trap is the average spectrum of the individual product ion spectra of all possible kinetic energies.

Figure 3 shows that the maximum ion kinetic energy increases approximately quadratically with the amplitude of the applied dipole field if the other parameters are fixed. This increase agrees with the expectation of eq 8 for a pseudo harmonic oscillator. Furthermore, Figure 3 implies that the maximum amount of kinetic energy deposited in the precursor ion can be controlled by adjusting the amplitude of the dc dipole field.

\section{Gas Chromatography Tandem Mass Spectrometry}

The combination of gas chromatography and tandem mass spectrometry in the ion trap is complicated by the change in ion secular frequency with ion density. The number of ions within the trap may vary several orders during the elution of chromatographic peaks. As the number of ions in the trap increases, the ion secular frequency shifts to lower frequency, which results in a variation in the amount of energy deposited to the precursor ion by resonance excitation and in changes of the product ion yields [17]. An octopole component in the trapping field will cause additional changes in the secular frequency of the ion because the frequency will become a function of the ion amplitude [35]. Nonresonance excitation is a mass

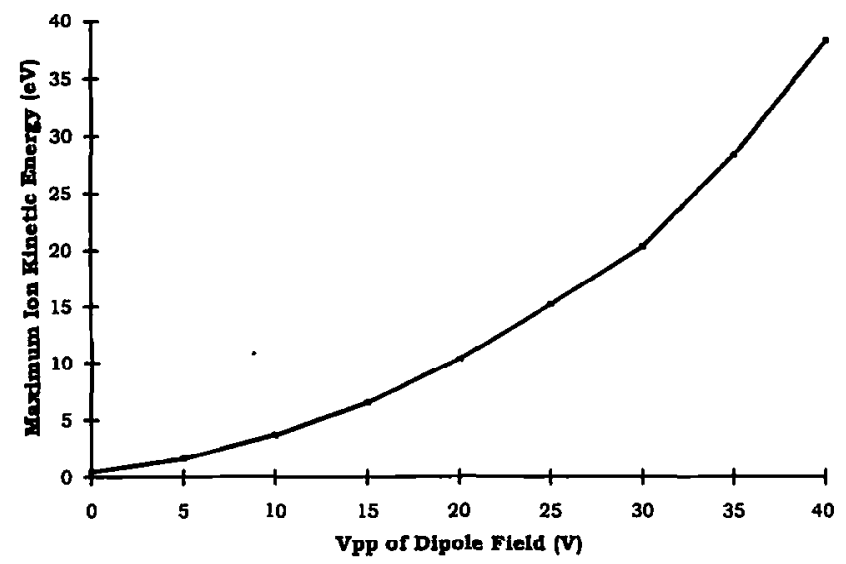

Figure 3. Maximum ion kinetic energy deposited by nonresonance excitation at different amplitudes of the applied dipole field. 
independent excitation method and the excitation is not affected by secular frequency changes. Thus, the energy deposited to the precursor ions and, therefore, the product ion spectra, remain unchanged even when the ion density in the trap varies. This characteristic is useful for GC-MS/MS applications where the sample concentrations quickly change and frequency calibrations would be difficult.

Figure 4 shows the gas chromatography-mass spectrometry (GC-MS) reconstructed single ion chromatogram for $100 \mathrm{pg}$ of $n$-butylbenzene and the corresponding GC-MS/MS ion chromatogram by using nonresonance excitation with a fixed ionization time. The CID process in the GC-MS/MS chromatogram is achieved by applying a $100-\mathrm{Hz} 42-\mathrm{V}_{\mathrm{p}-\mathrm{p}}$ square wave at an rf trapping voltage of $528 \mathrm{~V}_{0-\mathrm{p}}$ for $10 \mathrm{~ms}$. The signal-to-noise ratio $(\mathrm{S} / \mathrm{N})$ of 3624 in the GC-MS/MS ion chromatogram (Figure $4 \mathrm{a}$ ) is more than an order of

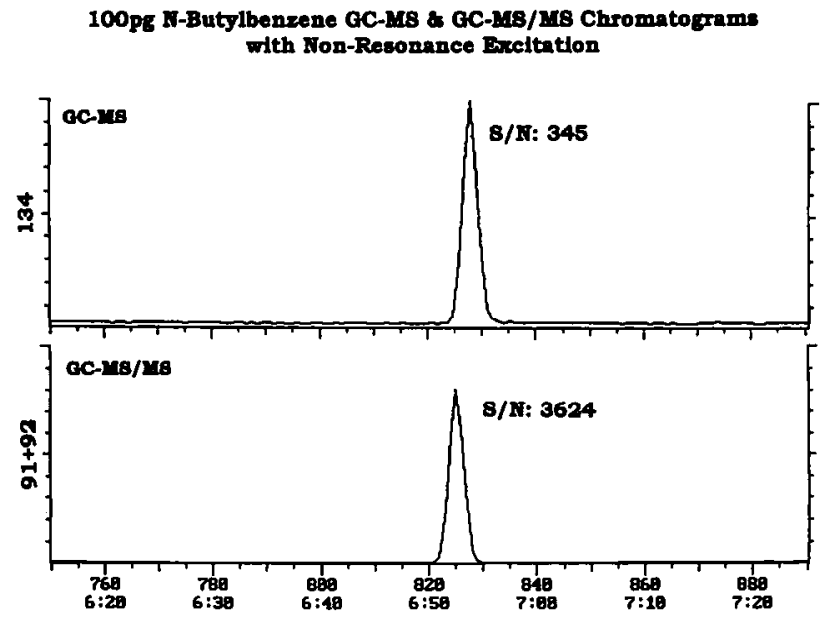

(a)

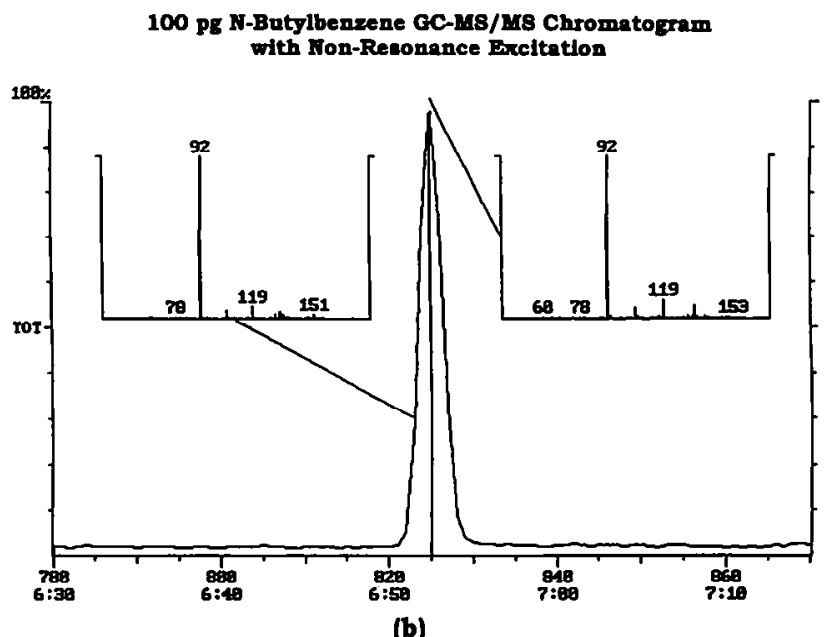

Figure 4. (a) Ion chromatograms of 100-pg $n$-butylbenzene GCMS (upper) and GC-MS/MS (lower) by using nonresonance excitation. (b) The expanded GC-MS/MS total ion chromatogram. The tandem mass spectra remain unchanged when the sample concentration, thus ion density, varies across the GC peak from the base to the apex when nonresonance excitation is used. magnitude higher than the $S / N$ (345) of the 100-pg $n$-butylbenzene GC-MS ion chromatogram acquired under the same gas chromatography (GC) and mass spectrometry conditions. This improvement in $\mathrm{S} / \mathrm{N}$ is due to background reduction, an increased ionization time, and the high efficiency of collision-induced dissociation [15] in the ion trap. Yost and co-workers [17] reported that product ions of $m / z 91$ and 92 of $n$ butylbenzene could be observed over the entire GCMS / MS chromatographic peak with broadband excitation (10-kHz bandwidth). The expanded GC-MS/MS chromatogram in Figure $4 \mathrm{~b}$ shows that the tandem mass spectrometry product ion spectra of $n$-butylbenzene from the base to the apex of the GC peak by using nonresonance excitation remain unchanged, even when the sample concentration (and therefore ion density) varies from base to apex of the GC peak.

The tetraethylsilane fragmentation pathway and its activation energies have been reported previously [13] as

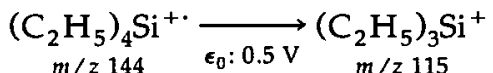

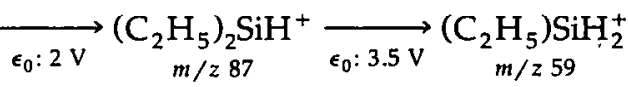

In this work, this compound is used to study the relationship between internal energy deposition and the amplitude of the square wave. Molecular ions of tetraethylsilane first are isolated and then are excited for $5 \mathrm{~ms}$ at an $\mathrm{rf}$ trapping voltage of $490 \mathrm{~V}_{0-\mathrm{p}}$. Nonresonance excitation used a $500-\mathrm{Hz}$ square wave differentially applied to the two endcaps. Resonance excitation used a single frequency sine wave with trapping field modulation as described previously [15]. The breakdown curves in Figure 5a show the effect on the product ion intensity of variation of the square-wave amplitude for nonresonance excitation. It is evident that the precursor ion is the dominant peak while the dc dipole is off. The intensity of the product ion $\left(\mathrm{C}_{2} \mathrm{H}_{5}\right)_{3} \mathrm{Si}^{+}$increases with an increase of the dc dipole voltage because more precursor ions gain enough internal energy to fragment. The intensity of this ion reaches its maximum when the amplitude of the $\mathrm{dc}$ dipole is about $28 \mathrm{~V}_{\mathrm{p}-\mathrm{p}}$. At higher voltages the intensity of the ion $\left(\mathrm{C}_{2} \mathrm{H}_{5}\right)_{3} \mathrm{Si}^{+}$decreases and the intensity of each of the other ions increases and then decreases. Figure 5a also shows that with nonresonance excitation, the precursor and each product ion appear in different square-wave amplitude ranges. The breakdown curve for resonance excitation is shown in Figure $5 b$ as a comparison. In contrast to nonresonance excitation, all product ions appear in a similar amplitude range of the ac excitation voltage. In addition, product ion yields are reduced significantly with increasing ac voltages.

Because the amplitude of the square wave is the only parameter that requires adjustment in nonresonance excitation, the optimization of CID parameters 


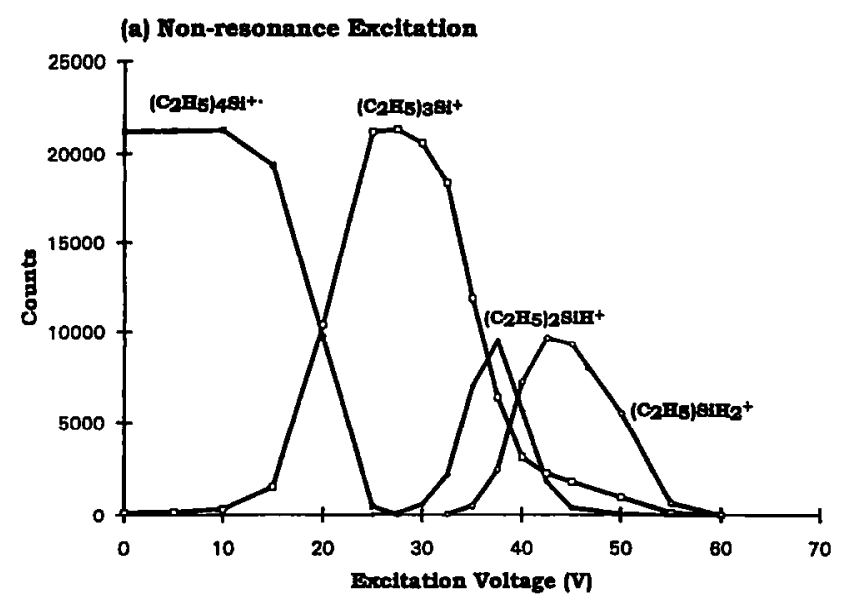

(b) Resonance Inxcitation

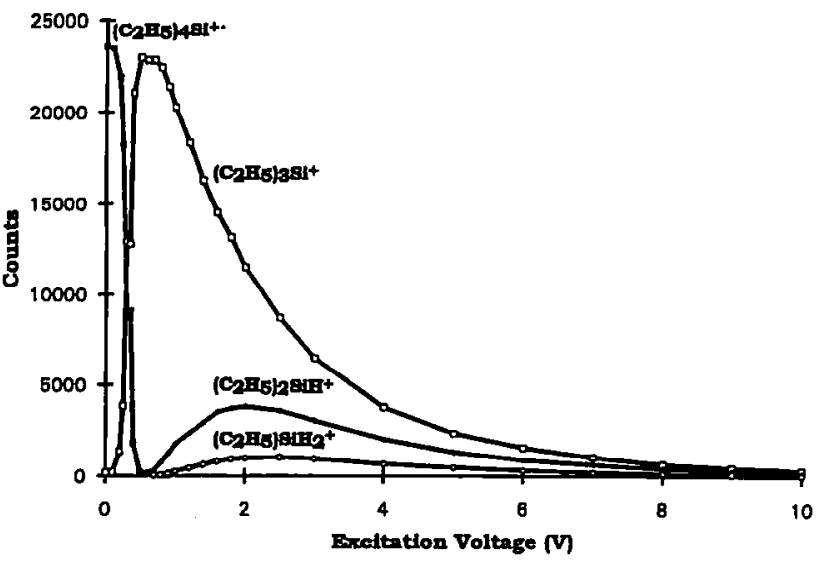

Figure 5. Breakdown curves of tetraethylsilane. (a) Nonresonance excitation. (b) Resonance excitation.

for nonresonance is simpler than for resonance excitation, in which both frequency and amplitude of the excitation waveform need to be optimized. Because nonresonance excitation is a slowly varing function of mass (see eq 14), it can be used to cause CID in precursor ions of similar mass, for example, to excite both analyte and its coeluting isotopically labeled internal standard for GC-MS/MS analysis.

\section{Selected Ejection Chemical Ionization}

Cairns et al. [32] have shown that ion trap chemical ionization (CI) spectra may be complicated by the presence of ions that originate from the electron ionization (EI) of the analyte, which results in a combined EI and CI spectrum. Insertion of a time period between the ionization and reaction periods, in which the sample fragment ions from EI are selectively ejected, produces a chemical ionization spectrum without the contamination of ions from electron ionization. Usually, chemical reagent gases have ions whose masses are lower by a specified value (less than $\sim 50 \mathrm{u}$ ) than the masses of sample EI fragment ions (greater than $50 \mathrm{u}$ ). Therefore, a CI spectrum without contamination of sample EI fragment ions may be obtained by applica- tion of a low pass mass filter in the inserted period that ejects all ions with mass-to-charge ratios greater than $a$ specified value.

Figure 6 shows that, depending on the amplitude of the square wave, the ions with mass above $131 \mathrm{u}$ or with mass above $100 \mathrm{u}$ may be ejected selectively. In these experiments, a square wave with a frequency of $300 \mathrm{~Hz}$ is differentially applied to two endcaps for 5 ms. The amplitudes of the square wave are $20 \mathrm{~V}$ in the top figure and $33 \mathrm{~V}$ in the bottom figure. Nonresonance excitation under these conditions behaves like a low pass mass filter as described by eq 9 . Although it may not have unit mass resolution, the conditions are sufficient to allow its use in SECI to eject the unwanted sample EI fragments.

Figure 7 shows two ion trap $\mathrm{CI}$ scan functions. The top figure is the traditional CI scan function for an ion trap. During the ionization period, the rf level is lowered intentionally to reduce the storage of EI fragments from the sample. The bottom of Figure 7 is the SECI scan function. A period to eject sample EI fragments is inserted between the ionization and reaction periods. The ejection period typically is about $5-10 \mathrm{~ms}$. The rf values for the ionization, ejection, and reaction periods may be optimized individually. In high pressure CI and traditional ion trap low pressure $\mathrm{CI}$ ion sources, the EI fragments from the sample are only reduced and the resulting $\mathrm{CI}$ mass spectrum is still a combina-

\section{Non-Resonance as Low Pass Mass Filter}
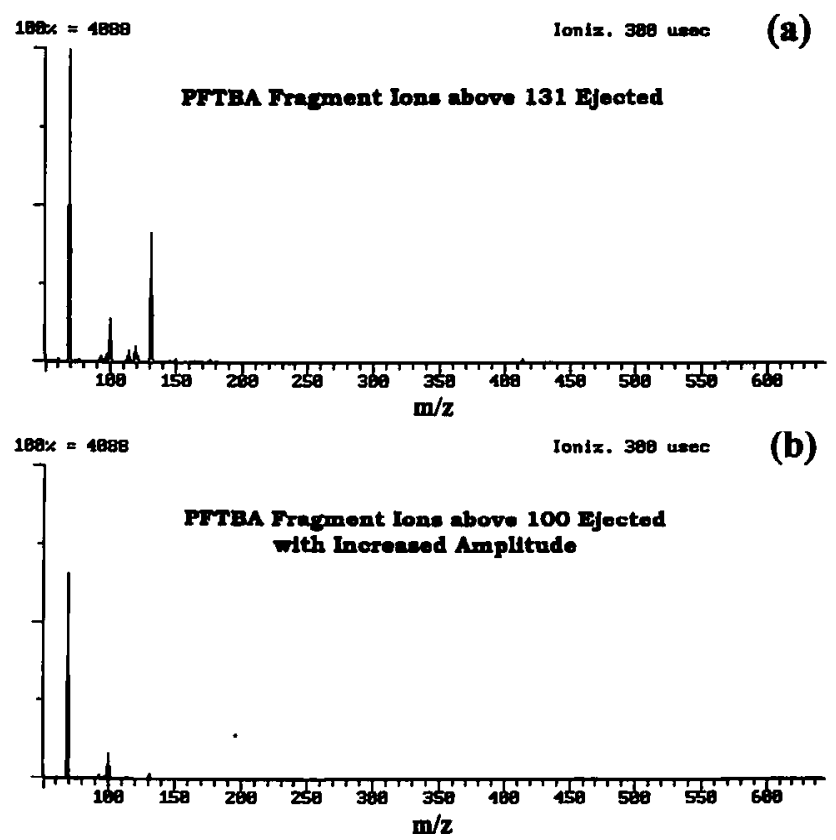

Figure 6. Nonresonance excitation used as a low pass mass filter. Dependent on the amplitude of the square wave, all ions with mass above $131 \mathrm{u}$ (upper) or with mass above $100 \mathrm{u}$ (bottom) may be ejected selectively. A $300-\mathrm{Hz}$ square is differentially applied to the two endcaps for $20 \mathrm{~ms}$. (a) The amplitude of the square wave is $20 \mathrm{~V}$. (b) The amplitude of the square wave is $33 \mathrm{~V}$. 


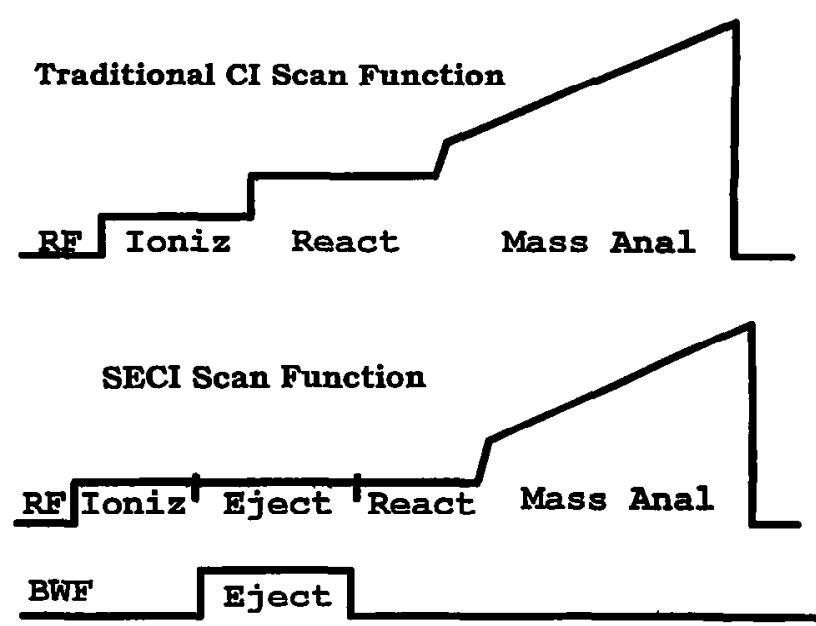

Figure 7. Ion trap CI scan functions. Top: The traditional CI scan function for an ion trap. During the ionization period, the if level is lowered to reduce the storage of EI fragments of the sample. Bottom: The SECI scan function. An EI fragment ejection period is inserted between the ionization and reaction periods. The rf values in the ionization, ejection, and reaction periods may be optimized individually.

tion of the CI and EI mass spectra. In the SECI mode, the EI fragments from the sample are eliminated totally and the resulting spectrum is a CI mass spectrum without the contamination of sample fragment ions from electron ionization.

The CI spectra of hexachlorobenzene are used to verify the improvement of the new SECI scan function. A T connector is placed inside the GC oven between the GC column and the transfer line into the ion trap. The solid hexachlorobenzene sample is placed in one of the ends of the $T$ connector and the hexachlorobenzene vapor is transported into the trap by the GC carrier gas (He). In the experiments, the GC oven is at $70^{\circ} \mathrm{C}$. Figure $8 \mathrm{a}$ is a spectrum acquired with the traditional CI scan function, in which the ratio of the molecular ion $\left[\mathrm{M}^{+}\right]$to the protonated molecular ion $[\mathrm{M}+1]^{+}$is $1: 2.5$. The $\mathrm{M}^{+} /[\mathrm{M}+1]^{+}$ratio approximately represents the EI/CI ratio [33]. The resulting hybrid spectrum cannot be assigned to any of the chlorine isotopic patterns. Figure $8 \mathrm{~b}$ is a spectrum acquired with the SECI scan function in which the protonated molecular ion cluster can be assigned unambiguously to a compound with six chlorine atoms and can be matched with existing databases.

\section{Summary}

We have demonstrated a universal ion excitation and ejection method for quadrupole ion traps. In this method, ion energy deposition is achieved by the combination of the axial dc dipole and rf trapping fields. This method does not require the supplemental excitation frequency to match with the secular frequency of the precursor ions for CID and thus is free of the effects of secular frequency shifts that arise from

\section{Hexachlorobenzene CI Spectra}
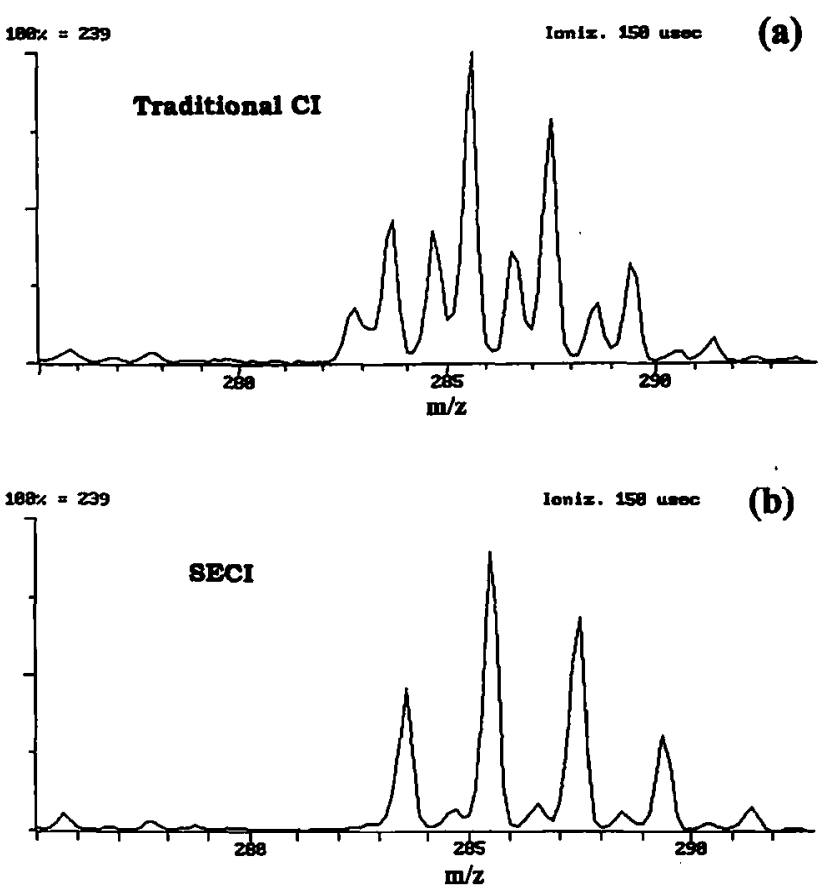

Figure 8. CI spectra of hexachlorobenzene. (a) A spectrum acquired with the traditional $\mathrm{CI}$ scan function, in which the ratio of the molecular ion $\mathrm{M}^{+}$to the protonated molecular ion $[\mathrm{M}+1]^{+}$, is 1:2.5. (b) A spectrum acquired with the SECI scan function.

space-charge. This lack of frequency dependence simplifies the optimization of CID parameters. This method can be used to excite multiple precursor ions simultaneously and is useful for GC-MS/MS analysis with a coeluting isotopically labeled internal standard. Simulations show that nonresonance excitation provides an effective means to quickly increase the precursor ion kinetic energy that may facilitate the study of precursor ions with multiple decomposition channels. It has been shown also that the nonresonance excitation method, used as a low pass mass filter, can be used in selective ejection chemical ionization to produce a $\mathrm{CI}$ spectrum without contamination of sample fragment ions from electron ionization.

\section{Acknowledgments}

We wish to thank Edward Marquette for his design of the modified electronic circuits and for helpful discussions.

\section{References}

1. Strife, R. J.; Kelley, P. E.; Weber-Grabau, M. Rapid Commun. Mass Spectrom. 1988, 2, 105.

2. Kaiser, R. E., Jr.; Louris, J. N.; Amy, J. W.; Cooks, R. G. Rapid Commun. Mass Spectrom. 1989, 3, 225.

3. Kaiser, R. E., Jr.; Cooks, R. G.; Stafford, G. C., Jr.; Syka, J. E. P.; Hemberger, P. H. Int. J. Mass Spectrom. Ion Processes 1991, 106, 79. 
4. Strife, R. J.; Simms, J. R. Int. J. Mass Spectrom. Ion Processes $1993,124,1$

5. Louris, J. N.; Amy, J. W.; Ridley, T. Y.; Cooks, R. G. Int. J. Mass Spectrom. Ion Processes 1989, 88, 97.

6. Van Berkel, G. J.; Glish, G. L.; McLuckey, S. A. Anal. Chem. 1990, 62, 1284.

7. Pedder, R. E.; Yost, R. A.; Weber-Grabau, M. Proceedings of the 37th Conference on Mass Spectrometry and Allied Topics; Miami Beach, FL, 1989; $p 468$.

8. Lin, H. Y.; Voyksner, R. D. Rapid Commun. Mass Spectrom. 1994, 8, 333.

9. Schwartz, J. C.; Syka, J. E. P.; Jardine, I. J. Am. Soc. Mass Spectrom. 1991, 2, 198.

10. Williams, J. D.; Cooks, R. G. Rapid Commun. Mass Spectrom. 1992, 6, 524

11. Goeringer, D. E.; McLuckey, S. A.; Glish, G. A. Proceedings of the 39th Conference on Mass Spectrometry and Allied Topics; Nashville, TN, 1991; p 1481.

12. Londry, F. A.; Wells, G. J.; March, R. E. Rapid Commun. Mass Spectrom. 1993, 7, 43.

13. Louris, J. N.; Cooks, R. G.; Syka, J. E. P.; Kelley, P. E.; Stafford, G. C., Jr.; Todd, J. F. J. Anal. Chem. 1987, 59, 1677.

14. McLuckey, S. A.; Glish, G. L.; Van Berkel, G. J. Int. I. Mass Spectrom. Ion Processes 1991, 106, 213.

15. Bolton, B.; Wells, G. J.; Wang, M. Proceedings of the 41st Conference on Mass Spectrometry and Allied Topics; San Francisco, CA, 1993; p 474a.

16. Todd, J. F. J.; Penman, A. D.; Thorner, D. A.; Smith, R. D. Rapid Commun. Mass Spectrom. 1990, 4, 108.

17. Yates, N. A.; Yost, R. A.; Bradshaw, S. C.; Tucker, D. B. Proceedings of the 39th Conference on Mass Spectrometry and Allied Topics; Nashville, TN, 1991; p 132.

18. Marshall, A. G.; Wang, T.-C. L.; Ricca, T. L. U.S. Patent $4,761,545,1988$.
19. Julian, R. K., Jr.; Cooks, R. G. Anal. Chem. 1993, 65, 1827.

20. Vedel, F.; Vedel, M.; March, R. E. Int. J. Mass Spectrom. Ion Processes 1991, 108, R11.

21. McLuckey, S. A.; Goeringer, D. E.; Glish, G. L. Anal. Chem. 1992, 64, 1455.

22. Curcuruto, O.; Fontana, S.; Traldi, P.; Celon, E. Rapid Commun. Mass Spectrom. 1992, 6, 322.

23. Liere, P.; Blasco, T.; March, R. E.; Tabet, J.-C. Rapid Commun. Mass Spectrom. 1994, 8, 953.

24. Lammert, S. A.; Cooks, R. G. Rapid Commun. Mass Spectrom. 1992, 6, 528.

25. Cooks, R. G.; Cleven, C. D.; Horn, R. A.; Nappi, M.; Weil, C.; Soni, M. H.; Julian, R. K., Jr. Int. J. Mass Spectrom. Ion Processes 1995, $146 / 147,147$.

26. Wang, M.; Wells, G. J. Proceedings of the 41st Conference on Mass Spectrometry and Allied Topics; San Francisco, CA, 1993; p 463a.

27. Wells, G. J.; Wang, M. U.S. Patent 5,381,006, 1995.

28. Wuerker, R. F.; Shelton, H.; Langmuir, R. V. J. Appl. Phys. $1959,30,342$.

29. Schachterle, S. Proceedings of the 42nd Conference on Mass Spectrometry and Allied Topics; Chicago, IL, 1994; p 714.

30. Brodbelt, J. S.; Louris, J. N.; Cooks, R. G. Anal. Chem. 1987, $59,1278$.

31. Louris, J. N.; Syka, J. E. P.; Kelley, P. E. U.S. Patent 4,686,367, 1987.

32. Cairns, T.; Chiu, K. S.; Siegmund, E. Rapid Commun. Mass Spectrom. 1992, 6, 331

33. Cairns, T.; Chiu, K. S.; Siegmund, E.; Weber, M. Rapid Commun. Mass Spectrom. 1992, 6, 449.

34. Dahl, D. A.; Delmore, J. E. SIMION PC/PS2 Version 4.0; Idaho National Engineering Laboratory, April 1988.

35. Franzen, J. Int. I. Mass Spectrom. Ion Processes 1991, 106, 63. 